through linkage of multiple national data sources. BMC Infect Dis. 2015;15:551. https://doi.org/10.1186/ s12879-015-1247-7

7. Public Health England. Research and analysis: national COVID-19 surveillance reports: GOV.UK; 2021 [cited 2020 Oct 2]. https://www.gov.uk/government/publications/ national-covid-19-surveillance-reports

8. Ladhani SN, Flood JS, Ramsay ME, Campbell H, Gray SJ, Kaczmarski EB, et al. Invasive meningococcal disease in England and Wales: implications for the introduction of new vaccines. Vaccine. 2012;30:3710-6. https:/ / doi.org/10.1016/ j.vaccine.2012.03.011

9. Taha MK, Deghmane AE. Impact of COVID-19 pandemic and the lockdown on invasive meningococcal disease. BMC Res Notes. 2020;13:399. https:/ / doi.org/10.1186/ s13104-020-05241-9

Addresses for correspondence: Shamez Ladhani or Sathyavani Subbarao, Immunisation and Countermeasures Division, Public Health England, 61 Colindale Ave, London NW9 5EQ, UK; email: shamez.ladhani@phe.gov.uk or vani.subbarao@phe.gov.uk

\section{SARS-CoV-2 Infection among Pregnant and Postpartum Women, Kenya, 2020-2021}

\author{
Nancy A. Otieno, ${ }^{1}$ Eduardo Azziz-Baumgartner, ${ }^{1}$ \\ Bryan O. Nyawanda, Eunice Oreri, Sascha Ellington, \\ Clayton Onyango, Gideon O. Emukule \\ Author affiliations: Kenya Medical Research Institute, Kisumu, \\ Kenya (N.A. Otieno, B.O. Nyawanda); Centers for Disease \\ Control and Prevention, Atlanta, Georgia, USA \\ (E. Azziz-Baumgartner, S. Ellington); Ministry of Health, Siaya, \\ Kenya (E. Oreri); Centers for Disease Control and Prevention, \\ Kisumu, Kenya (C. Onyango); Centers for Disease Control and \\ Prevention, Nairobi, Kenya (G.O. Emukule)
}

DOI: https://doi.org/10.3201/eid2709.210849

We determined incidence of severe acute respiratory syndrome coronavirus 2 and influenza virus infections among pregnant and postpartum women and their infants in Kenya during 2020-2021. Incidence of severe acute respiratory syndrome coronavirus 2 was highest among pregnant women, followed by postpartum women and infants. No influenza virus infections were identified.

${ }^{1}$ These authors contributed equally to this article.
Tnformation about the incidence of severe acute respiratory syndrome coronavirus 2 (SARSCoV-2) infection among hospitalized pregnant women is available (1), but information about incidence among pregnant women in the community is not. We therefore quantified the incidence of symptomatic laboratory-confirmed SARS-CoV-2 and influenza infections among pregnant and postpartum women and their infants in Kenya during 2020-2021. The study was reviewed and approved by the Kenya Medical Research Institute Scientific and Ethics Review Unit (KEMRI SSC. 2880) and the Centers for Disease Control and Prevention (CDC) Institutional Review Board (CDC protocol 6709; 45 C.F.R. part 46; 21 C.F.R. part 56). All participants provided written consent.

We adapted an ongoing prospective multiyear influenza mother/baby cohort to include SARSCoV-2 testing (2). Pregnant women at $<31$ weeks of gestation who were seeking prenatal care in Siaya County, Kenya, were approached for enrollment. Those who provided informed consent completed a survey about their demographics and antenatal history and were tested for HIV infection. Women were then phoned or visited at home once weekly until delivery and through their postpartum period, together with their infants, for 6 months to

\begin{tabular}{|c|c|}
\hline \multicolumn{2}{|c|}{$\begin{array}{l}\text { Table. Characteristics of pregnant and postpartum women and } \\
\text { their infants with laboratory-confirmed severe acute respiratory } \\
\text { syndrome coronavirus } 2 \text { infection, Kenya, May 2020-February } \\
2021^{*}\end{array}$} \\
\hline Characteristic & Values \\
\hline \multicolumn{2}{|l|}{ Women, $\mathrm{n}=16$} \\
\hline Days from onset to swabbing, mean (SD) & $2.6(1.9)$ \\
\hline \multicolumn{2}{|l|}{ Care-seeking from onset, d } \\
\hline$<2$ & $11(68.8)$ \\
\hline$>2$ & $5(31.3)$ \\
\hline \multicolumn{2}{|l|}{ Self-reported symptoms } \\
\hline Fever in past $48 \mathrm{~h}$ & $2(12.5)$ \\
\hline Measured fever $>38.0^{\circ} \mathrm{C}$ & $2(12.5)$ \\
\hline Cough & $16(100)$ \\
\hline Shortness of breath & $1(6.3)$ \\
\hline Runny nose & $10(62.5)$ \\
\hline Headache & $10(62.5)$ \\
\hline Muscle/ joint pain & $2(12.5)$ \\
\hline Antimicrobial medication & $16(100)$ \\
\hline \multicolumn{2}{|l|}{ Infants, $\mathrm{n}=2$} \\
\hline Days from onset to swabbing, mean (SD) & $2.5(2.1)$ \\
\hline \multicolumn{2}{|l|}{ Care-seeking from onset, $d$} \\
\hline$\leq 2$ & $1(50.0)$ \\
\hline$>2$ & $1(50.0)$ \\
\hline \multicolumn{2}{|l|}{ Clinical signs reported by mother } \\
\hline Fever in previous $48 \mathrm{~h}$ & $1(50.0)$ \\
\hline Measured fever $\geq 38.0^{\circ} \mathrm{C}$ & $1(50.0)$ \\
\hline Cough & $2(100)$ \\
\hline Runny nose & $1(50.0)$ \\
\hline Diarrhea & $1(50.0)$ \\
\hline Antimicrobial medication & $2(100)$ \\
\hline
\end{tabular}




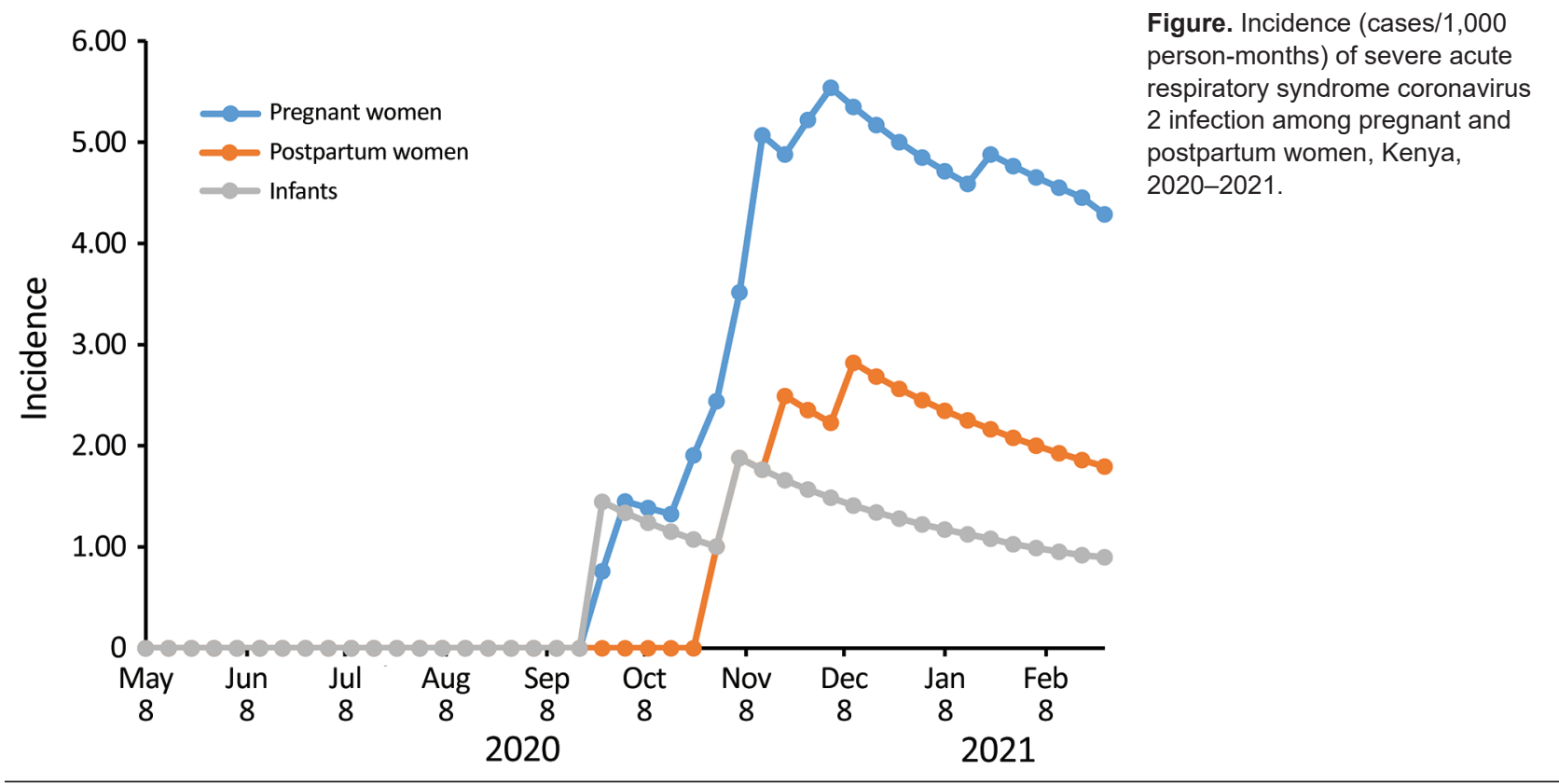

identify coronavirus disease (COVID-19)-like illness (CLI) as defined by CDC (3). Those reporting CLI underwent nasopharyngeal and oropharyngeal swabbing at study clinics. Specimens were tested for SARS-CoV-2 and influenza viruses by real-time reverse transcription PCR at the KEMRI laboratory in Kisumu, Kenya.

During May 2020-February 2021, KEMRI staff approached 1,056 pregnant or postpartum women and enrolled 1,023 (97\%). Half of enrolled women had primary school education, and $40 \%$ ran small businesses. A total of 180 (18\%) were HIV infected, of which 177 (98\%) were receiving antiretroviral medication. A total of $116(11 \%)$ were vaccinated against influenza. Each of 3 women had hypertension, diabetes mellitus, or tuberculosis.

As of February 2021, staff had followed 886 pregnant women, who contributed 2,786 personmonths, and 695 postpartum women and infants, who contributed 2,264 person-months (some women were represented in both groups). CLI developed in 274 (31\%) pregnant women (348 episodes), $133(19 \%)$ postpartum women (162 episodes), and $231(33 \%)$ infants (277 episodes). Swab samples were collected within $\leq 10$ days of illness from $58 \%$; positive SARS-CoV-2 results were obtained for $12 / 200(6 \%)$ pregnant women, 4/100 (4\%) postpartum women, and 2/200 (1\%) infants. None had positive influenza virus test results. The most common clinical manifestations of COVID-19 among pregnant and postpartum women were cough $(16 / 16 ; 100 \%)$, runny nose $(10 / 16 ; 63 \%)$ and head- ache $(10 / 16 ; 63 \%)$. Cough was identified for each of the 2 SARS-CoV-2-infected infants (Table). The rate of SARS-CoV-2 infection rapidly increased during follow-up. In the population tested, the cumulative incidence of SARS-CoV-2 infection per 1,000 person-months was 4.3 (pregnant women), 1.8 (postpartum women), and 0.9 (infants) (Figure).

CLI occurred in $19 \%-33 \%$ of participants, of which a small percentage had laboratory-confirmed SARS-CoV-2 infection. The incidence of SARS-CoV-2 infection in this population, however, was rapidly rising during the study period. No influenza viruses were identifiable during the historic influenza epidemic period (4). SARS-CoV-2 rates seemed higher among pregnant women, then postpartum women, and lowest among infants.

A study limitation is our inability to exhaustively assess symptoms of CLI among infants (e.g., headache, sore throat, loss of taste and smell) because we relied on the mothers' reports. This limitation would potentially underestimate the burden of COVID-19 among infants. In addition, we did not quantify asymptomatic and mildly symptomatic infections that might have been missed. However, we plan to test acute-phase and convalescent-phase serum, cord blood, and placentas to identify asymptomatic infections and explore whether risk for SARS-CoV-2 infection truly differs.

In summary, our findings suggest a higher burden of COVID-19 during pregnancy. These results highlight the potential benefit of prioritizing COVID-19 vaccination for pregnant women. 
Financial support for conducting the research was provided by the Influenza Division, National Center for Immunization and Respiratory Diseases, CDC (grant no. 5U01GH002133-04-00). CDC also participated in the design of the study, analysis and interpretation of data, writing of the report, and decision to submit the article for publication.

\section{About the Author}

Ms. Otieno is an assistant principal research scientist at the Kenya Medical Research Institute, Centre for Global Health Research. Her primary research interests are infectious diseases, specifically maternal and child health.

\section{References}

1. Zambrano LD, Ellington S, Strid P, Galang RR, Oduyebo T, Tong VT, et al.; CDC COVID-19 Response Pregnancy and Infant Linked Outcomes Team. Update: characteristics of symptomatic women of reproductive age with laboratoryconfirmed SARS-CoV-2 infection by pregnancy status United States, January 22-October 3, 2020. MMWR Morb Mortal Wkly Rep. 2020;69:1641-7. https:/ / doi.org/10.15585/ mmwr.mm6944e3

2. Nyawanda BO, Otieno NA, Otieno MO, Emukule GO, Bigogo G, Onyango CO, et al. The impact of maternal human immunodeficiency virus infection on the burden of respiratory syncytial virus among pregnant women and their infants, Western Kenya. J Infect Dis. 2020 Aug 10 [Epub ahead of print].

3. Centers for Disease Control and Prevention. National Notifiable Diseases Surveillance System: surveillance case definitions. Coronavirus disease 2019 (COVID-19) 2020 interim case definition, approved April 5, 2020 [cited 2021 Feb 24]. https:// wwwn.cdc.gov/nndss/conditions/ coronavirus-disease-2019-covid-19/case-definition/2020

4. Olsen SJ, Azziz-Baumgartner E, Budd AP, Brammer L, Sullivan S, Pineda RF, et al. Decreased influenza activity during the COVID-19 pandemic - United States, Australia, Chile, and South Africa, 2020. MMWR Morb Mortal Wkly Rep. 2020;69:1305-9. https://doi.org/10.15585/mmwr. mm6937a6

5. Flannery DD, Puopolo KM. Perinatal COVID-19: guideline development, implementation, and challenges. Curr Opin Pediatr. 2021;33:188-94. https://doi.org/10.1097/ MOP.0000000000000997

Address for correspondence: Nancy A. Otieno, Kenya Medical Research Institute, Centre for Global Health Research, PO Box 1578-40100, Kisumu, Kenya; email: notieno@kemricdc.org, nancyotieno@gmail.com.

\section{Genomic Evolution of SARS-CoV-2 Virus in Immunocompromised Patient, Ireland}

\author{
Maureen Lynch, ${ }^{1}$ Guerrino Macori, ${ }^{1}$ \\ Séamus Fanning, Edel O'Regan, Eoin Hunt, \\ Dermot O'Callaghan, Brian McCullagh, \\ Cormac Jennings, Anne Fortune
}

Author affiliations: Mater Misericordiae University Hospital, Dublin, Ireland (M. Lynch, E. O’Regan, E. Hunt, D. O’Callaghan,

B. McCullagh, C. Jennings, A. Fortune); University College

Dublin-Centre for Food Safety School of Public Health,

Physiotherapy \& Sports Science, Dublin (G. Macori, S. Fanning)

DOI: https://doi.org/10.3201/eid2709.211159

We examined virus genomic evolution in an immunocompromised patient with prolonged severe acute respiratory syndrome coronavirus 2 infection. Genomic sequencing revealed genetic variation during infection: 3 intrahost mutations and possible superinfection with a second strain of the virus. Prolonged infection in immunocompromised patients may lead to emergence of new virus variants.

$\mathrm{T}$ The coronavirus disease (COVID-19) pandemic, caused by severe acute respiratory syndrome coronavirus 2 (SARS-CoV-2), has led to substantial illness and death in immunocompromised patients (1). Outcomes for patients with hematologic malignancies can be poor because of immune suppression associated with cancer itself and chemoimmunotherapy regimens used to treat these cancers (2).

Persistent shedding of SARS-CoV-2 RNA has been described since early in the pandemic; quantitative reverse transcription PCR (qRT-PCR) results have remained positive for 63 days (3). Recent studies of immunocompromised patients have detected infectious virus until 143 days after diagnosis (4-6). Phylogenetic analysis showed that single-nucleotide polymorphisms (SNPs) could be used to elucidate the transmission routes of SARS-CoV-2 in communities (7). Moreover, it has been demonstrated that intrahost single-nucleotide variants are restricted to specific lineages (8); however, no clear evidence supports a link between prolonged infection and intraevolutionary dynamics (9).

We report a case of a prolonged clinical infection with persistent virus shedding in a patient with functional B-cell deficiency, hypogammaglobulinemia,

${ }^{1}$ These authors contributed equally to this article. 